\title{
The horizon line, linear perspective, interposition, and background brightness as determinants of the magnitude of the pictorial moon illusion
}

\author{
Stephanie A. H. Jones ANd AleXander E. Wilson \\ University of New Brunswick, Saint John, New Brunswick, Canada
}

\begin{abstract}
A total of 110 undergraduate students participated in a series of three experiments that explored the magnitude of the moon illusion in pictures. Experiment 1 examined the role of the number and salience of depth cues and background brightness. Experiment 2 examined the role of the horizon line, linear perspective, interposition, and background brightness. In Experiment 3, comparative distance judgments of the moon as a function of linear perspective, interposition, and the size of the standard moon were obtained. The magnitude of the moon illusion increased as a function of the number and salience of depth cues and changes in background brightness. Experiment 2 failed to support the role of the horizon line in affecting the illusion. Experiment 3 provided additional support for the illusory distance component of the moon illusion.
\end{abstract}

The moon illusion is the apparent overestimation of the size of the horizon moon when compared with the zenith moon and/or an apparent underestimation of the distance of the horizon moon when compared with the zenith moon. In reality, there is no significant difference in the distance from the earth to the moon as a function of the moon's position in the sky. As such, if perception is veridical, the observer should perceive the moon's size and distance to be constant.

Past research has revealed great magnitude differences between explorations of the natural moon illusion and drawings of the moon embedded in various contexts. However, images, similar to drawings that have been previously used to display optical illusions (e.g., the Ponzo illusion or the Müller-Lyer illusion), have been shown to be a viable means through which to examine this illusion (see, e.g., Coren \& Aks, 1990; Redding, 2002). The present study incorporated the use of computer-generated drawings of the moon embedded in several different contexts. These drawings were adapted from Coren and Aks (1990; see Figure 1 below). It is important to note that the term elevated moon will be used to refer to a drawing of a moon in an elevated position in a two-dimensional plane, and the term zenith moon will be used to refer to the real moon viewed at optical infinity when it takes its position in the zenith of the sky.

In a series of experiments that sought to explore the magnitude of the moon illusion in drawings, Coren and Aks (1990) found that, in the presence of pictorial distance cues, the horizon moon was judged to be larger than the elevated moon overall. Participants selected a moon that they perceived to match in size to the moon in each experimental drawing from a series of comparison moons. Each drawing was rated on its overall impression of depth by several professors at the University of British Columbia. Participant overestimations of the horizon moon, when compared with the elevated moon, were found to increase as the number of depth cues in the drawings increased (see Coren \& Aks, 1990, for a full review). Similar results were found in a follow-up experiment in which participants were asked to indicate the size of the horizon and elevated moons using the method of reproduction (i.e., reproducing the perceived diameter of the experimental moon by marking on a line). Results indicated that the horizon moon was perceived to be $11 \%$ larger than the elevated moon in the drawings rated highest in their overall impression of depth, and only $6 \%$ larger in the drawings that were rated the lowest in their overall impression of depth.

Overall, these results suggest that an increase in the number of distance cues in a picture is associated with a greater perceived size difference between a moon in the horizon position and a moon in an elevated position. In addition, apart from magnitude differences when compared with the exploration of the natural moon illusion, the role of depth cues in the magnitude of the illusion as reported by Coren and Aks (1990) is fairly consistent with research in field settings using the real moon, and in laboratory experiments using artificial moons (see, e.g., Kaufman \& Rock, 1962; Rock \& Kaufman, 1962). However, the suggested role of apparent distance in the overestimation of the size of the horizon moon as compared with the zenith moon has been contradicted by the size-distance paradox.

According to the concept of size constancy, the moon in its horizon position - in addition to being perceived as larger - should be perceived as being farther away than the moon in its elevated position. However, the majority of viewers perceive the natural moon at the horizon to be closer than the moon in its zenith position (Boring, 1943). 
A

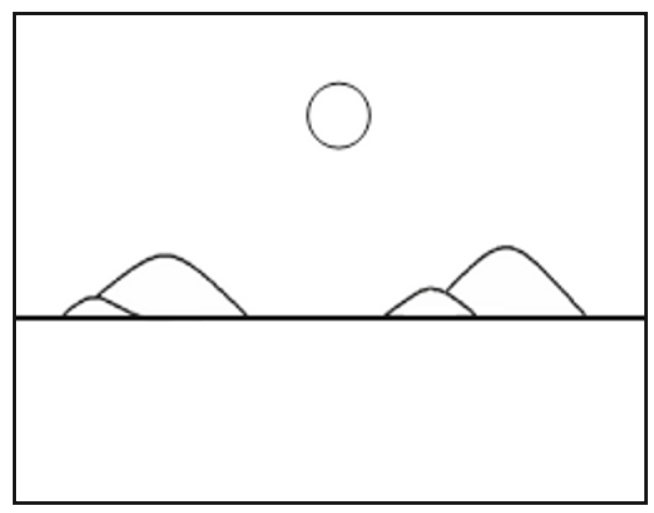

C

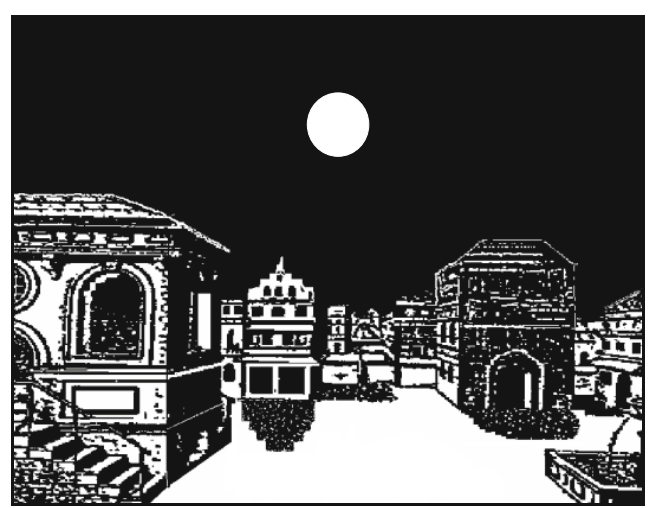

B

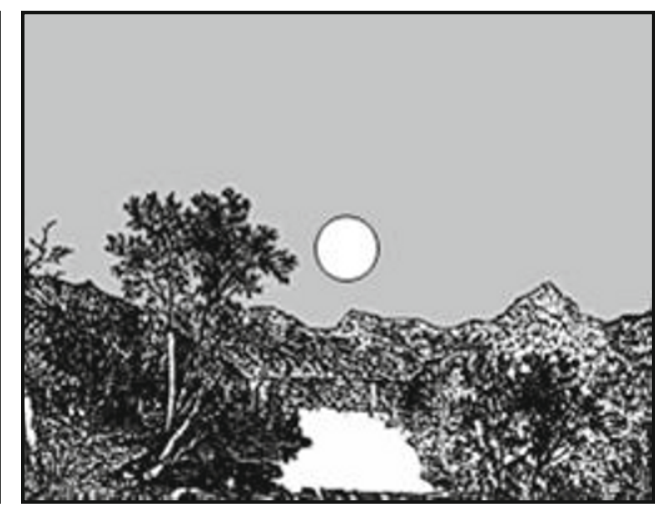

D

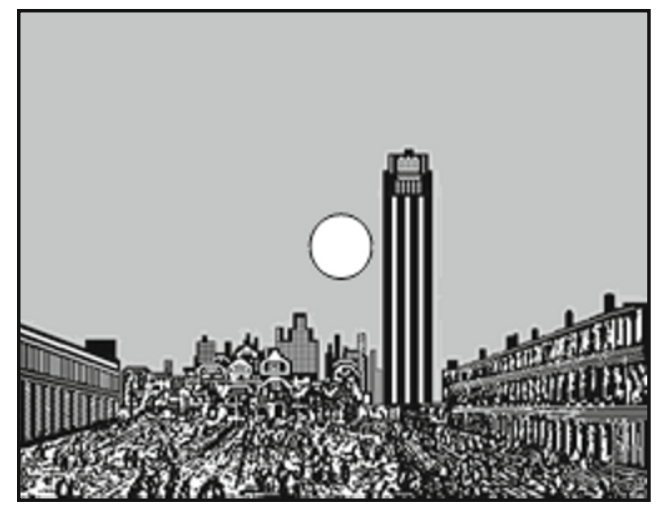

Figure 1. Examples of several computer-generated drawings: (A) Drawing rated the lowest in depth cue salience. (B) Drawing rated as intermediate in depth cue salience. (C) Drawing rated high in depth cue salience-town scene. (D) Drawing rated high in depth cue salience-city scene. From "Moon Illusion in Pictures: A Multimechanism Approach," by S. Coren and D. J. Aks, 1990, Journal of Experimental Psychology: Human Perception \& Performance, 16, p. 367. Copyright 1990 by the American Psychological Association. Adapted with permission.

This phenomenon has also been found in the exploration of the moon illusion in drawings. For example, Coren and Aks (1990) found that when participants were asked to make a comparative distance judgment between a horizon and an elevated moon embedded in the same context, participants judged the horizon moon to be closer than the elevated moon overall, regardless of the salience of the depth cues in the drawing.

Although it could be suggested that a bias effect contributed to the results of this study (i.e., differences in apparent size contributed to the judgment of distance), Coren and Aks (1990) reexamined the illusory distance component of the illusion in drawings using only a moon in a horizon position. They paired high-depth cue salience drawings with low-depth cue salience drawings and asked participants to indicate which of the horizon moons appeared to be closer. Results indicated that $70 \%$ of participants judged the horizon moon in the high-depth cue salience drawing to be closer than the horizon moon in the low-depth cue salience drawings

The primary objective of the present study was to explore how the magnitude of the moon illusion in drawings would vary as a function of manipulations in background brightness, the number of distance cues in a drawing, and the type of distance cues in a drawing (specifically, the horizon line, interposition, and linear perspective). Similarly, an exploration of the apparent distance of the experimental moon as a function of the type of distance cue and the size of the moon stimulus was of primary interest.

Because the horizon line cannot be removed when exploring the natural moon illusion, it is very difficult to separate the role of the horizon line in the magnitude of this illusion. However, past research has suggested that a detailed exploration of the role of the horizon line may prove useful. Kaufman and Rock (1962) reported an almost doubling of the illusory effects when the natural moon was viewed over the large expanse of the ocean as compared with when it was viewed over a city skyline-a circumstance in which a horizon line did not create a clear distinction between the sky and terrain.

In addition, although research has suggested that apparent brightness may play a role in apparent size (see, e.g., Holway \& Boring, 1940; Robinson, 1954), this relationship has not been explored in relation to the moon illusion 
in drawings. Experiments 1 and 2 of the present study included a manipulation of background brightness through the use of white, gray, and black backgrounds.

Lastly, speculation concerning the relationship between apparent size and apparent distance has been fueled by the size-distance paradox (Hershenson, 1989). This illusory distance component of the moon illusion has not been explored as extensively in drawings as has the illusory size component. Similarly, the role of standard moon size-or the variation in comparative distance judgments as a function of moon stimulus size - has yet to be examined. Since the size-distance paradox is based on the relationship between apparent size and apparent distance, an exploration of the apparent distance of the moon in drawings should include a manipulation of the size of the standard moon.

In summary, Experiment 1 examined the role of the number and salience of depth cues and background brightness in the magnitude of the illusion. Experiment 2 examined the role of the horizon line, linear perspective, interposition, and background brightness. In Experiment 3, comparative distance judgments of the moon as a function of (1) linear perspective and interposition and (2) the size of the standard moon were obtained.

\section{EXPERIMENT 1}

\section{Method}

\section{Participants}

Thirty participants - 26 females and 4 males, ages 17 to 35 years $(M=19.4, S D=3.82)$ - participated in Experiment 1. All participants had normal or corrected-to-normal vision and were naive to the purpose of the experiment.

\section{Materials}

The stimuli for Experiment 1 included four computer-generated drawings adapted from Coren and Aks (1990; Figure 1). These drawings included two high-depth cue salience drawings, one intermediatedepth cue salience drawing, and one low-depth cue salience drawing. The level of depth cue salience was determined by ranking each drawing on its overall impression of depth on a 5-point Likert scale, ranging from 1 (no impression of depth) to 5 (a strong impression of depth). In addition, all four drawings were ranked on the presence of six depth cues (texture gradient, interposition, height in plane, linear perspective, familiar size, and shadowing) on a 5-point Likert scale, ranging from 1 (not present) to 5 (definitely present). The rankings were completed by two graduate students and two professors in the psychology department at the University of New Brunswick, Saint John, and were comparable to the ratings as reported by Coren and Aks (1990).

Background brightness was manipulated by creating a white, gray, and black background version for each of the four drawings. Each context possessed a horizon (in proximity to the horizon line division) and an elevated moon version. These will be referred to generally as the two moon positions and the results will be discussed in terms of the horizon moon or the elevated moon. Six control stimuli were used to establish a baseline measure of apparent size. These stimuli included drawings of the moon in its horizon and elevated positions for each of the three levels of background brightness (Figure 2). The addition of the control stimuli to the four experimental contexts, three background brightness levels, and two moon positions resulted in a total of 30 experimental drawings. In all conditions, the moon was white.

All drawings were displayed using an LCD projector. The presentation of the drawings and recording of each participant response was controlled by E-Prime software (Psychology Software Tools Inc., Pittsburgh, PA). In each trial, the standard moon in each experimental drawing was $61 \mathrm{~mm}$ in diameter (subtended visual angle $=2.18^{\circ}$ ) and was presented on the left-hand side of the projection screen. When the moon was in a horizon position, it appeared $20.7 \mathrm{~cm}$ from the bottom of the computer-displayed drawing, and the elevated moon appeared $26.5 \mathrm{~cm}$ above the bottom of the drawing. The angular separation between the horizon and elevated moons was $13.46^{\circ}$. The comparison moons included 11 white circles (outlined in black), ranging in size from $54.9 \mathrm{~mm}$ to $65.88 \mathrm{~mm}$ (increasing in increments of $1.22 \mathrm{~mm}$; range of angular size: $2.13^{\circ}$ to $2.40^{\circ}$ ), presented on a white empty field. Four different sequences of comparison moons were used. These sequences included 10 (of the 11) comparison moons presented from smallest to largest horizontally in two lines on the right-hand side of the projection screen. The smallest circle was omitted from Sequence 1, the largest circle was omitted from Sequence 2, and the two smallest and two largest circles were omitted from Sequences 3 and 4, respectively. All drawings (experimental and comparison) were $56 \mathrm{~cm}$ high and $46 \mathrm{~cm}$ wide, once projected.

\section{Procedure}

Participants were seated in a chair placed $160 \mathrm{~cm}$ from the projection screen. The projector was placed behind and slightly to the left of participants' heads and was raised $119 \mathrm{~cm}$ above the floor to ensure that all stimuli were presented at eye level, that the participant would not obstruct the light beam, and that there was no glare produced on the displayed drawings. Each of the four sequences of comparison moons was paired with each of the 30 experimental drawings, and E-Prime presented the stimulus pairs randomly in each block and to each participant. Each participant also completed the entire experiment twice, resulting in a total of 240 size comparisons. In each trial, participants were asked to make a comparative size judgment by selecting from among the comparison moons that which they perceived to most closely match in size to the standard moon in each experimental drawing. Participants indicated their selection by pressing the number assigned to their choice comparison moon on a wireless keyboard, which was set on a table in front of them. To limit position effects, each trial was followed by a blank screen.

\section{Results}

The number and salience of depth cues, as well as background brightness, were manipulated in Experiment 1. Error judgments were calculated by subtracting the correct circle response $(C)$ from each participant response $(P R)$ for each stimulus. The computational formula was $P R-$ $C=$ Error . The correct circle response was the comparison moon in each trial that was the same linear diameter as the moon in the experimental image for that trial. As such, an error score consisted of the difference between the linear diameter of the correct circle response in a particular trial and the linear diameter of the circle selected by the participant in that trial. Positive error values indicated an overestimation in the size of the stimulus moon, and negative error values indicated an underestimation in the size of the stimulus moon.

Analysis included a repeated measures ANOVA with error scores as the dependent variable. Significant differences among the means following planned comparisons were determined through the use of a one-tailed paired samples $t$ test with the significance level set at .05 . Figure 3 provides an overview of the mean error scores broken down by moon position and drawing.

\section{Main Effects}

Position. The analysis revealed a significant difference in apparent size judgments between the moon in the ho- 
A

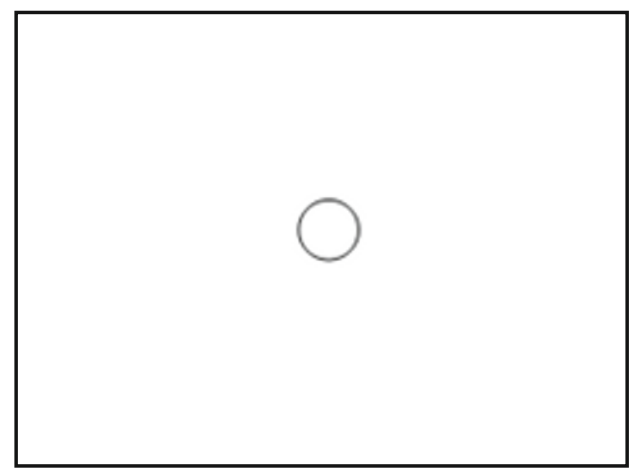

B

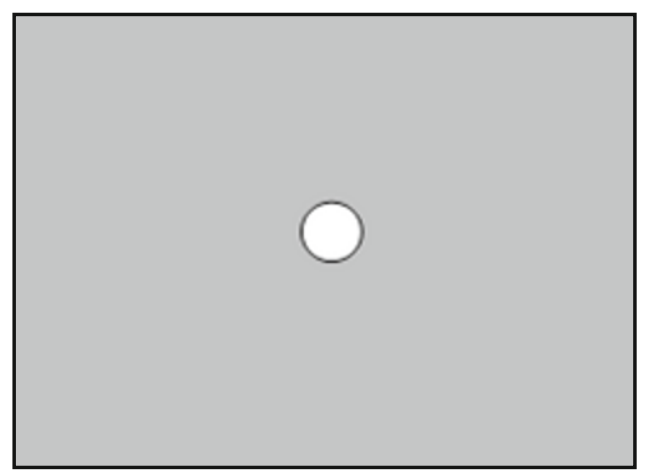

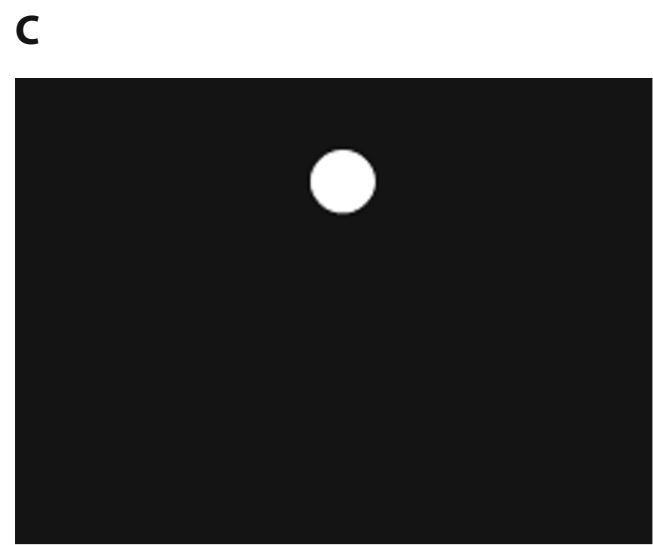

Figure 2. Three of the six computer-generated control drawings used to form a baseline measure of apparent size in Experiments 1 and 2.

rizon position (located in proximity to the horizon line division in each drawing) and the moon in its elevated position $\left[F(1,29)=21.33, p<.01, \eta_{\mathrm{p}}^{2}=.42\right]$. Participants judged the moon in its horizon position to be larger $(M=$ $61.04 \mathrm{~mm}, S D=.21 \mathrm{~mm}$ ) overall than the moon in its elevated position $(M=60.55 \mathrm{~mm}, S D=.24 \mathrm{~mm})$.

Background. A significant difference in size judgments was also found among the three background brightness conditions: white, gray, and black $[F(2,58)=17.55$, $\left.p<.01, \eta_{\mathrm{p}}^{2}=.37\right]$. All planned comparisons revealed significant differences $(p<.05)$. Overall, participants judged the moon to be larger in the black background condition $(M=61.26 \mathrm{~mm}, S D=.25)$ than in the gray $(M=$ $60.66 \mathrm{~mm}, S D=.21 \mathrm{~mm})$ or the white $(M=60.46 \mathrm{~mm}$, $S D=.21 \mathrm{~mm}$ ) background condition.

Drawing. A significant main effect of drawing was found $\left[F(4,116)=72.90, p<.01, \eta_{\mathrm{p}}^{2}=.71\right]$. Planned comparisons revealed significant differences in participant size judgments between the control drawing and all four experimental drawings $(p<.05)$, and also as a result of all other comparisons $(p<.05)$. Overall, as the number and salience of depth cues in the drawings increased, participants' size judgments also increased; the moon was perceived to be largest in the two drawings rated highest in depth cue salience $(M=61.51 \mathrm{~mm}, S D=.24 \mathrm{~mm} ; M=$ $61.78 \mathrm{~mm}, S D=.25 \mathrm{~mm}$, respectively) and smallest in the control conditions $(M=59.33 \mathrm{~mm}, S D=.26 \mathrm{~mm})$.
Comparison sequence. The analysis revealed a significant difference between comparison sequences $\left[F(3,87)=175.46, p<.01, \eta_{\mathrm{p}}^{2}=.86\right]$. Post hoc pairwise comparisons revealed significant differences among all comparison sequences $(p<.01)$. Overall, participants judged the standard moon to be larger when paired with those comparison sequences from which smaller circles were omitted $(M=61.11 \mathrm{~mm}, S D=.23 \mathrm{~mm} ; M=$ $61.91 \mathrm{~mm}, S D=.22 \mathrm{~mm}$ ) than when paired with those comparison sequences from which larger circles were omitted $(M=60.24 \mathrm{~mm}, S D=.24 \mathrm{~mm} ; M=59.93 \mathrm{~mm}$, $S D=.25 \mathrm{~mm})$. However, no significant interactions were found between comparison sequence and any other factor explored. As such, the role of comparison sequence did not interfere with any of the factors initially hypothesized to play a role in participants' judgments of size.

\section{Interactions}

Position and drawing. The analysis also revealed a significant interaction between moon position and type of drawing $\left[F(4,116)=32.81, p<.01, \eta_{\mathrm{p}}^{2}=.53\right]$. The effect of depth cues on apparent size was larger for the horizon moon than for the elevated moon (Figure 3). This effect seems to be particularly prominent in the high-depth-cue-salience town-scene and high-depth-cue-salience city-scene conditions. Similarly, the elevated moon was judged to be larger than the horizon moon in the control condition. However, 


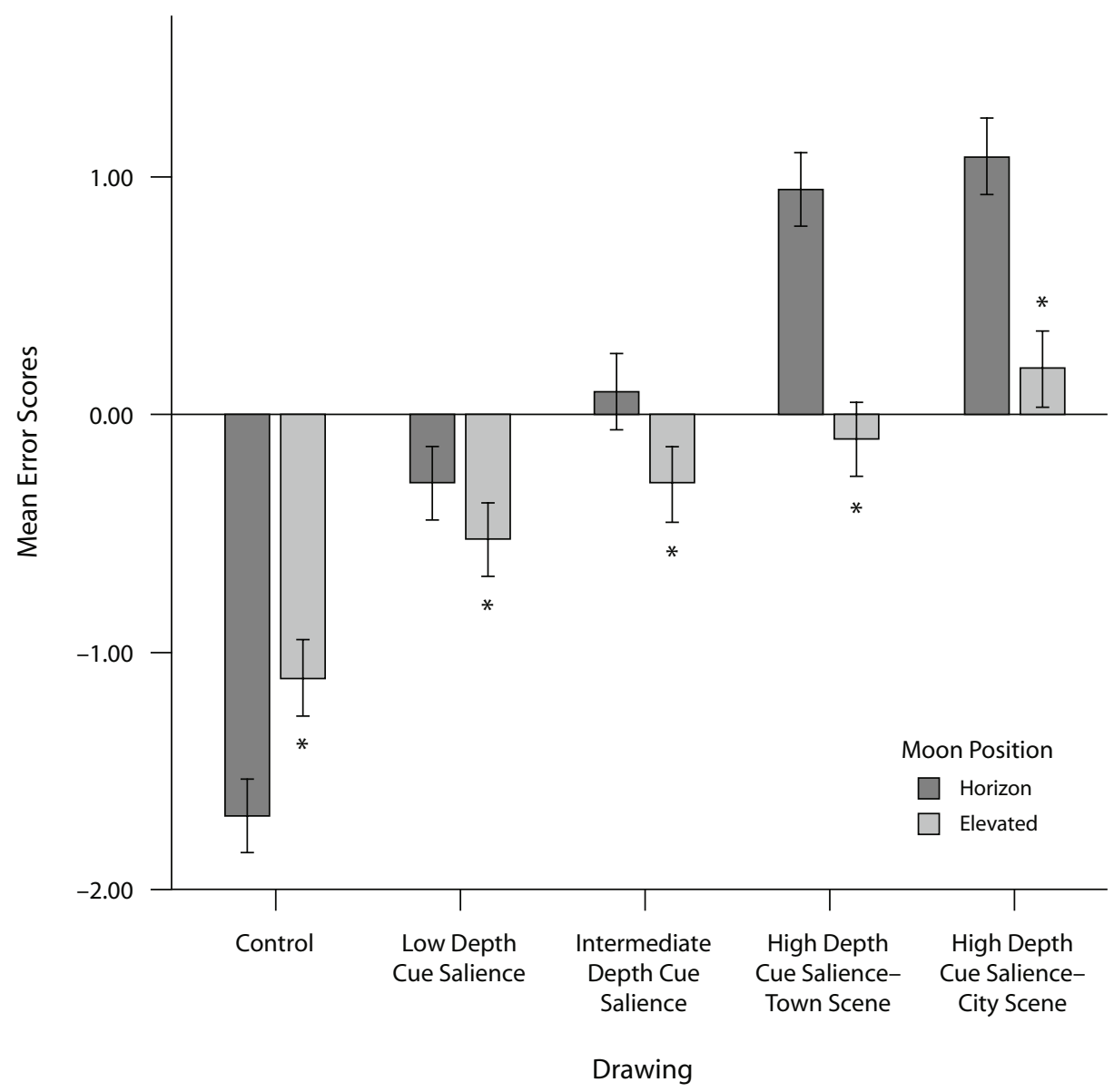

Figure 3. Experiment 1: Mean error scores broken down by moon position and type of drawing. The * indicates a significant difference between the horizon and elevated moon. Error bars display the $95 \%$ confidence interval for each condition.

with the addition of cues to distance, the horizon moon was overestimated in comparison with the elevated moon.

\section{Discussion}

The horizon moon was judged to be larger than the elevated moon overall in each of the four experimental drawings. Likewise, the addition of distance cues had a greater effect on the apparent size of the horizon moon than the elevated moon. In general, the horizon moon was perceived to be largest in the two drawings rated highest in depth cue salience.

The relationship between apparent size and apparent distance is a central consideration in the exploration of the moon illusion. Emmert's law-a specific variation of the size-distance invariance hypothesis (SDIH) - is often used as an explanation of the moon illusion. It suggests that "an object or image subtending a constant visual angle . . . will increase in perceived size as its perceived distance increases" (Hershenson, 1989, p. 236). Essentially, when we view the moon in its horizon position, we are presented with a number of distance cues from which we may infer size. However, when the moon is in its elevated position, these cues are not as prominent. In the case of a drawing, which provides two-dimensional information, we interpret the apparent distance of the horizon moon from the depth cues present in the image. As such, a greater number of distance cues should provide a greater impression of depth. The greater the perceived distance of the horizon moon, the larger we should perceive it to be.

Likewise, the adjacency principle suggests that the perception of object characteristics is a function of their position in space (Gogel, 1973). In the case of the moon illusion, the distance cues in the drawing may have a greater effect on the perception of the characteristics of the horizon moon because they are relatively close to one another. The elevated moon, on the other hand, is farther away from the distance cues present in the drawing, and thus, the distance cues have a smaller effect on the apparent size of the moon in that position.

However, in the case of the moon illusion, the sizedistance paradox has often been presented as a means to refute the SDIH or to suggest that it is not sufficient to explain the perceptual effects of the illusion. Size constancy states that we should perceive the horizon moon to be farther away in addition to being larger than the elevated moon, which is not always the case. Under the contention that the shape of the sky is often perceived as a flattened dome, King and Gruber (1962) explored the apparent size 
of after-images viewed using different parts of the sky as the viewing background (near the horizon and $45^{\circ}$ and $90^{\circ}$ elevated). Results indicated that the after-image viewed near the horizon was perceived to be larger than the afterimage viewed at the $45^{\circ}$-elevated (by $81 \%$ of participants) and $90^{\circ}$-elevated (by $87 \%$ of participants) positions.

As an explanation for the occurrence of the sizedistance paradox, Kaufman et al. (2007) suggested that we typically expect objects that are larger to be closer and, in doing so, tend to disregard distance cues that would suggest otherwise; therefore, the perceived distance of the moon may not be processed initially. Instead, we first decide on the size of the moon and infer distance. As evidenced by varying results, there remains a great deal of room for exploration and research concerning our biases toward size and distance estimation and comparisons.

Participants judged the moon to be larger overall in the black background conditions than in the white or gray background conditions. This finding has added ecological validity to the examination of the apparent size of the moon in drawings and is consistent with the documented relationship between apparent brightness and apparent size. For example, Robinson (1954) found that participants overestimated the size of light disks that were set at high luminance levels and underestimated the size of light disks that were set at low luminance levels. Likewise, since simultaneous contrast suggests that perception of the brightness or color of one area can be influenced by the brightness or color of adjacent areas (Goldstein, 2007), the black background may have affected the apparent brightness of the moon and, in turn, its apparent size.

The present study also highlights the importance of the use of proper control stimuli. In the case of the moon illusion in drawings, a control stimulus is required to assess the apparent size of the moon apart from any influence that additional cues may provide about size or distance. This type of measurement allows one to accurately conclude that the addition of a particular distance cue added to a drawing results in an increase, decrease, or no change in the magnitude of the illusion. Results indicated significant differences in the apparent size of the moon between the control image and all other images. In addition, the horizon moon was judged largest overall in the image rated highest in depth cue salience and smallest overall in the control image. Similarly, the elevated moon was judged to be larger than the horizon moon in the control condition. However, with the addition of distance cues, an overestimation of the size of the horizon moon in comparison with the size of the elevated moon was found.

Participants' judgments of size were found to vary significantly as a function of the type of comparison sequence. Participants judged the standard moon to be larger when paired with comparison sequences from which smaller moons were omitted than when compared with comparison sequences from which larger moons were omitted.

The central tendency effect posits that when making multiple perceptual judgments, participants tend to overestimate small magnitudes of a stimulus and to underestimate large magnitudes of a stimulus, thus directing their judgments toward a medium magnitude (Pressey, 1967). By contrast, in- termediate magnitudes of a stimulus do not tend to be overestimated or underestimated. The central tendency effect has been shown to be a common tendency in human behavior (Pressey, 1967), and it occurs throughout a variety of perceptual estimates, including judgments of distance, weight, and sound pitch (Helson, 1964). A central tendency effect in Experiment 1, combined with the omission of smaller circles in two of the comparison sequences, may have resulted in the participants' biasing their judgments toward overestimation of the standard moon size when paired with those two sequences. Essentially, the intermediate circles in the comparison sequences from which smaller circles were omitted would have been larger overall than the intermediate circles in sequences from which larger circles were omitted. However, it is important that we reiterate that no significant interactions were found between comparison sequence and any other factor initially hypothesized to play a role in the magnitude of this illusion.

\section{EXPERIMENT 2}

\section{Method}

\section{Participants}

Thirty-two participants-23 females and 9 males ages 17 to 46 years $(M=20.3, S D=5.97)$ - participated in Experiment 2. All participants had normal or corrected-to-normal vision and were naive to the purpose of the experiment.

\section{Materials}

The computer-generated experimental stimuli used in Experiment 2 included black-and-white drawings of the moon in a horizon or elevated position embedded in one of seven different contexts. These contexts were as follows: (1) horizon line; (2) linear perspective; (3) interposition; (4) horizon line and linear perspective; (5) horizon line and interposition; (6) linear perspective and interposition; and (7) horizon line, linear perspective, and interposition. Each drawing also included a white, gray, and black background version for each of the two moon positions (horizon and elevated; Figure 4). The same six control stimuli that were used in Experiment 1 were used in Experiment 2 to establish baseline measurements of apparent size. As such, a total of 48 experimental drawings were included in the experiment. Each experimental drawing was paired with each of the four sequences of comparison moons used in Experiment 1. This resulted in a set of 192 judgments. The entire set of judgments was completed twice by each participant. All images were presented to participants using the same procedure as that used in Experiment 1 . The method of selection, in which participants were asked to select a comparison moon that they perceived to match in size to the experimental moon in each trial, was once again used.

\section{Results}

In Experiment 2, we sought to examine the role of the horizon line, linear perspective, interposition, and background brightness in the magnitude of the illusory size component of the moon illusion in drawings. Error judgments were calculated by subtracting the correct circle response $(C)$ from each participant response $(P R)$ for each stimulus $(P R-$ $C=$ Error $)$. The correct circle response was the comparison moon in each trial that had the same linear diameter as the moon in the experimental image for that trial.

An error score thus consisted of the difference between the linear diameter of the correct circle response in a particular trial and the linear diameter of the circle selected by the participant in that trial. Positive error values indi- 
A

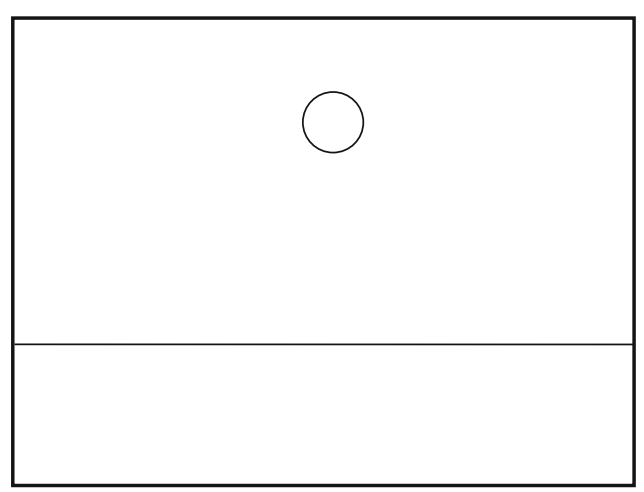

C

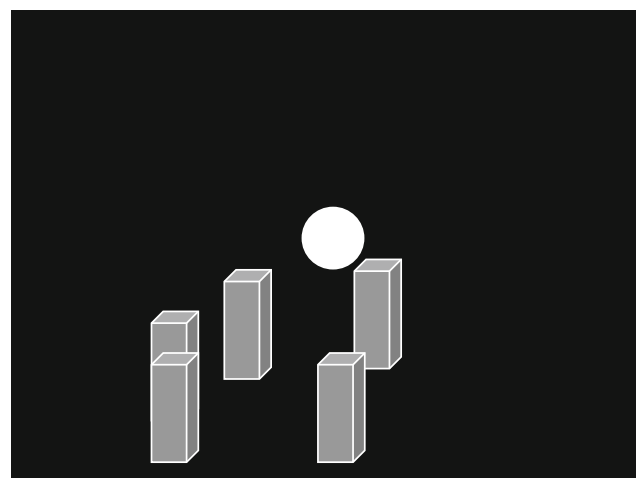

B

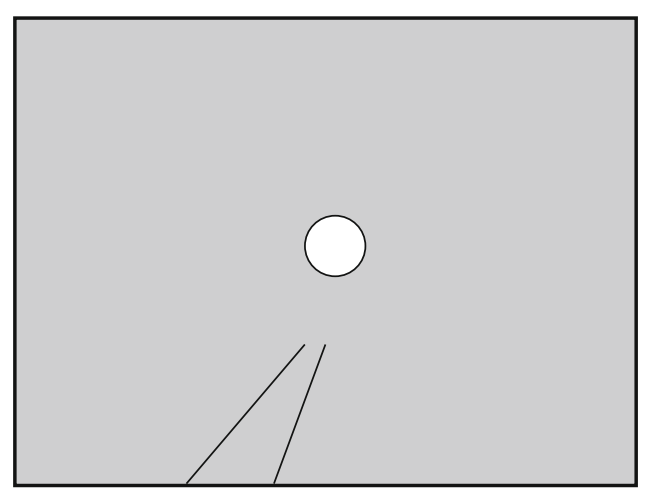

D

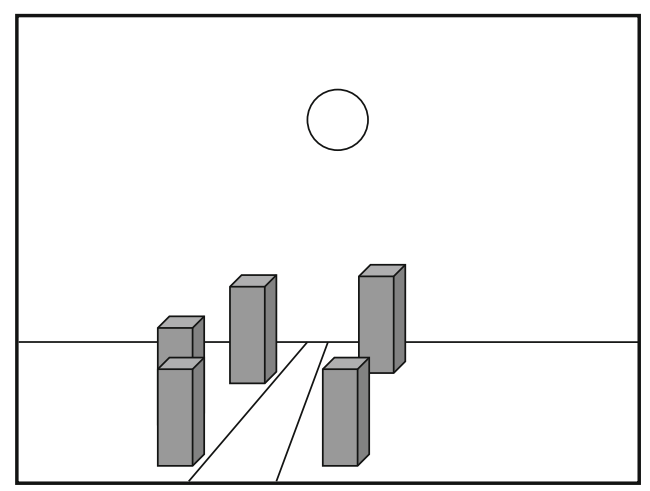

Figure 4. Sample of the experimental drawings presented to participants in Experiment 2. (A) Drawing containing the horizon line only on a white background. (B) Linear perspective only on a gray background. (C) Interposition only on a black background. (D) Horizon line, linear perspective, and interposition on a white background.

cated an overestimation in the size of the stimulus moon, and negative error values indicated an underestimation in the size of the stimulus moon. The analysis included a repeated measures ANOVA with error scores as the dependent variable. Significant differences among the means following planned comparisons were determined through the use of a one-tailed paired samples $t$ test, with the significance level set at .05 . Figures 5 and 6 provide an overview of the primary results of Experiment 2.

\section{Main Effects}

Position. The analysis revealed a significant main effect of moon position $\left[F(1,31)=8.42, p<.01, \eta_{\mathrm{p}}^{2}=.21\right]$. Participants judged the moon in its horizon position to be larger overall $(M=60.55 \mathrm{~mm}, S D=.29 \mathrm{~mm})$ than the moon in its elevated position $(M=60.27 \mathrm{~mm}, S D=.28 \mathrm{~mm})$.

Background. A significant difference in size judgments was also found among the three background brightness levels: white, gray, and black $[F(2,62)=4.45, p<$ $\left..05, \eta_{\mathrm{p}}^{2}=.13\right]$. Planned comparisons revealed significant differences in mean size judgments between the white and black background conditions and the gray and black background conditions. Overall, the apparent size of the moon was greater in the black background condition
$(M=60.58 \mathrm{~mm}, S D=.33 \mathrm{~mm})$ than in the gray $(M=$ $60.32 \mathrm{~mm}, S D=.27 \mathrm{~mm})$ or the white $(M=60.31 \mathrm{~mm}$, $S D=.27 \mathrm{~mm}$ ) background condition.

Drawing. A significant difference among drawings was found $\left[F(7,217)=12.27, p<.01, \eta_{\mathrm{p}}^{2}=.28\right]$. Planned comparisons revealed significant differences in participant size judgments between the control drawing $(M=59.95 \mathrm{~mm}$, $S D=.30 \mathrm{~mm})$ and all other images $(p<.05)$, except for the horizon-line-only image ( $M=60.06 \mathrm{~mm}, S D=.30 \mathrm{~mm})$. The apparent size of the moon increased as the number of distance cues in the drawing increased. The mean size judgment for the horizon line, linear perspective, and interposition condition was $60.68 \mathrm{~mm}(S D=.29 \mathrm{~mm})$.

Comparison sequence. The analysis revealed a significant difference between comparison sequences $\left[F(3,93)=265.52, p<.01, \eta_{\mathrm{p}}^{2}=.90\right]$. Post hoc pairwise comparisons revealed significant differences among all comparison sequences $(p<.01)$. Participants judged the standard moon to be larger overall when paired with those comparison sequences from which smaller circles were omitted $(M=60.79 \mathrm{~mm}, S D=.29 \mathrm{~mm} ; M=61.51 \mathrm{~mm}$, $S D=.27 \mathrm{~mm}$ ) than when paired with those comparison sequences from which larger circles were omitted $(M=$ $59.81 \mathrm{~mm}, S D=.30 \mathrm{~mm} ; M=59.51 \mathrm{~mm}, S D=.27 \mathrm{~mm}$ ). 


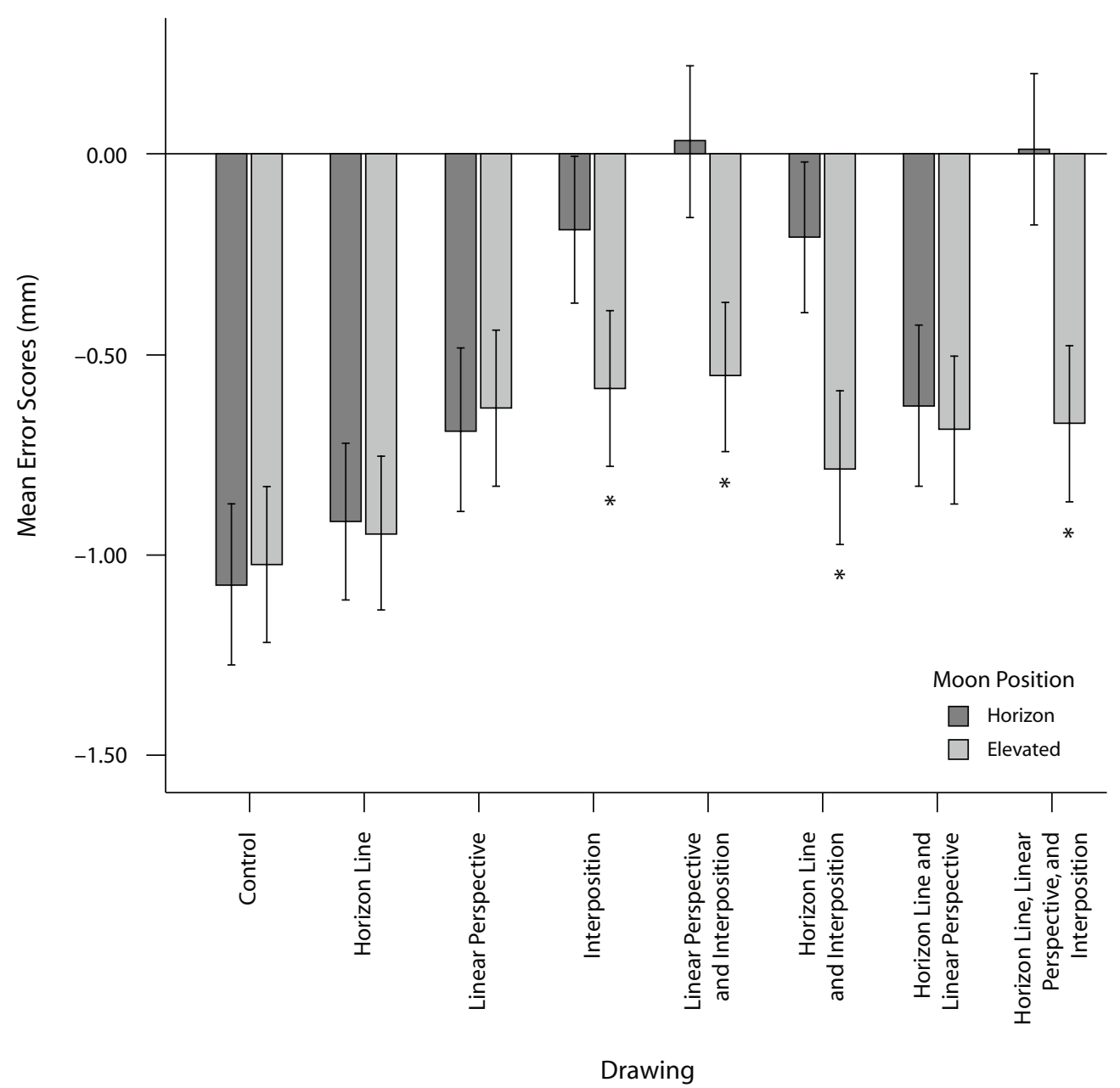

Figure 5. Mean error scores broken down by moon position and type of drawing. The * indicates a significant difference between the horizon and elevated moon. Error bars display the $95 \%$ confidence interval for each condition.

However, no significant interactions were found between comparison sequence and any other factor, suggesting that the effect of comparison sequence did not interfere with the effect of any other factor initially hypothesized to play a role in the magnitude of the illusion.

\section{Interactions}

Position and drawing. A significant interaction between moon position and type of drawing was found $\left[F(7,217)=8.20, p<.01, \eta_{\mathrm{p}}^{2}=.21\right.$; Figure 5]. The addition of subsequent distance cues had a greater effect on the apparent size of the horizon moon than on that of the elevated moon.

Background and drawing. A significant interaction was also found between background brightness and type of drawing $\left[F(14,434)=3.71, p<.05, \eta_{\mathrm{p}}^{2}=.11\right.$; Figure 6]. The differences between the control drawing and the images with linear perspective only, interposition only, and linear perspective and interposition together were greater in the black background condition than in the white or the gray background condition.

\section{Discussion}

Consistent with the results of Experiment 1, analyses revealed that the horizon moon was judged to be larger than the elevated moon overall. Similarly, the addition of depth cues had a greater effect on the apparent size of the horizon moon than the elevated moon. However, contrary to the initial hypothesis, size judgments did not appear to differ between moon positions as a function of the addition of a horizon line. In addition, it is also important that we address the size estimates overall. The reader may note that almost all apparent size judgments were underestimations when compared with the actual linear size of the moon. However, the moon illusion is defined as an overestimation of the horizon moon in comparison with the zenith moon. As such, the primary comparison when exploring this illusion is between the horizon and elevated moons.

In real-world contexts, the magnitude of the moon illusion is quite large when the moon is seen over flat expanses of land or ocean (see, e.g., Kaufman \& Rock, 1962; Rock \& Kaufman, 1962). However, in such circumstances, there remains a third dimension of depth regardless of the type 


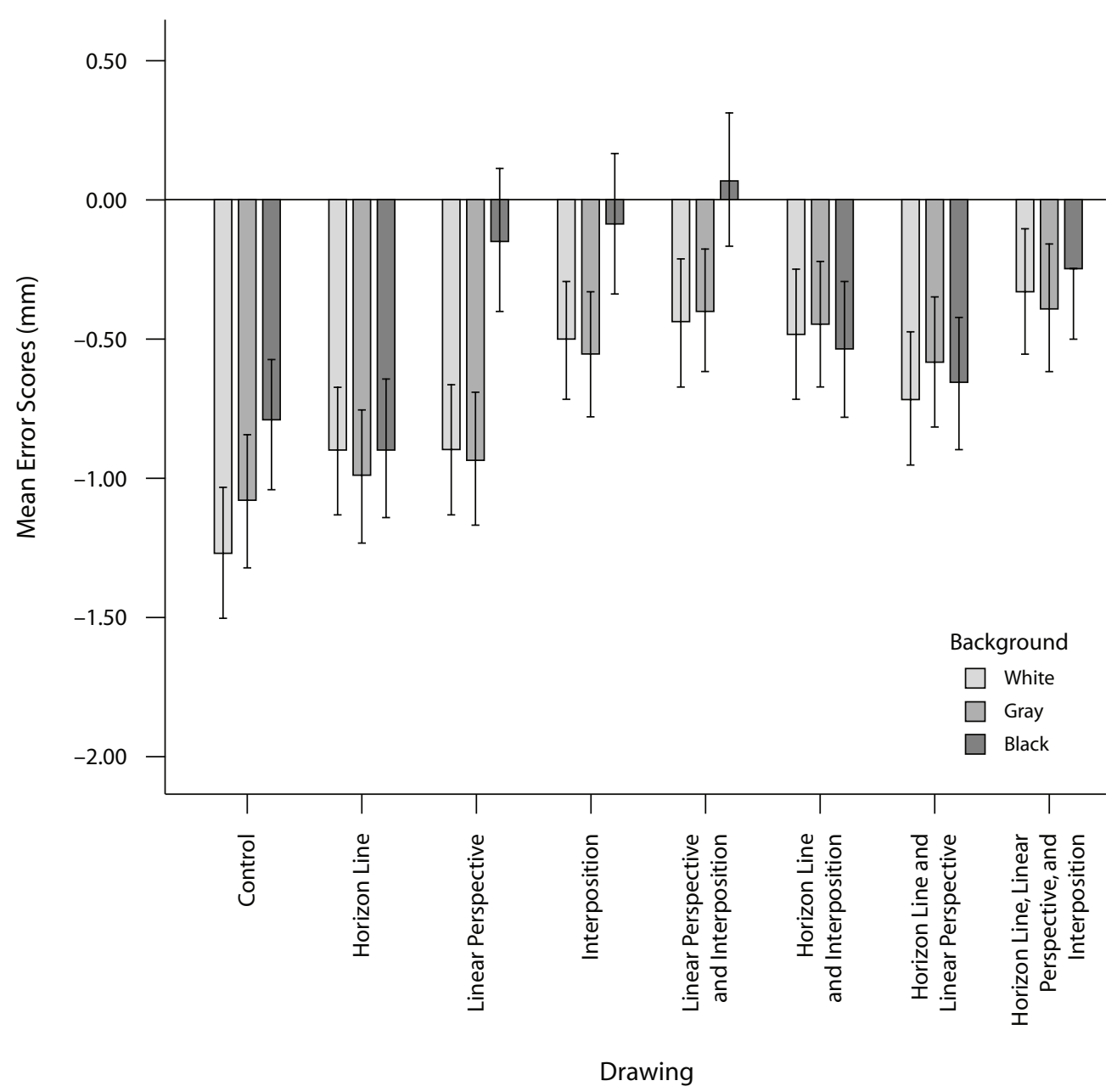

Figure 6. Mean error scores broken down by the type of background and the type of drawing. Error bars display the $95 \%$ confidence interval for each condition. Significant differences between groups are indicated by the nonoverlap of the confidence interval bars.

of cue available to the visual system. Similarly, the distance information provided by binocular disparity is a very important cue from which we infer distance in the real world, but it is not present when looking at a picture or drawing. In two-dimensional drawings, the third dimension is lost, and observers must rely on the specific distance cues in the image to infer size and/or distance. In fact, the apparent size of the horizon moon in comparison with the elevated moon in drawings tends to increase as the types of distance cues in the drawing - and the distances among them - vary (Enns, 2004). The addition of a horizon line to the drawings may not have provided enough distance information from which participants could infer the size of the moon.

Research has shown that the addition of interposition to an image can affect apparent size. Mandes (1985) presented children and adults with images of human figures and asked the participants to judge the size of each figure. Half of the images contained interposition, and half did not. Results indicated that the accuracy of size judgments increased as a result of the addition of interposition. The role of the horizon line, interposition, and linear perspective separately in the magnitude of the moon illusion in drawings had not previously been explored. The present study provides new findings concerning the effect of these specific pictorial distance cues.

Participants judged the moon to be larger, overall, in the black background conditions than in the white or gray background conditions, and the addition of subsequent distance cues had a greater effect on participant size judgments in the black background conditions than in the white and gray background conditions. This effect seemed to be particularly evident for the interposition, linear perspective, and interposition-and-linear-perspective-together conditions.

In exploring the relationship between apparent brightness, apparent distance, and apparent size, Over (1962) found that when a standard and a comparison stimulus were the same luminous intensity but subtended different visual angles, the object that subtended the smaller visual angle was consistently judged to be less bright than the object that subtended the larger visual angle. Since apparent brightness has been shown to influence the perception of both size and distance (see, e.g., Robinson, 1954), and the relationship between apparent size and apparent distance is well established in the literature (Hershenson, 1989), 
it becomes increasingly important that we examine the moderated relationship among all three of these factors. The moon illusion appears to be one such occurrence in which apparent brightness and apparent distance play a role in apparent size.

Participants judged the standard moon to be largest overall when it was paired with the two comparison sequences from which smaller moons were omitted. The central tendency effect may have once again contributed to this result. In addition, according to Watson (1957), when a participant is presented with a series of stimuli, such as in the case of the comparison sequences used in Experiments 1 and 2, the judgment of one stimulus in the series tends to be influenced by the other stimuli in the series. As such, if a comparison sequence with fewer small circles was presented first, participants may have judged those circles to be larger overall than the circles in the comparison sequence from which large circles were omitted. There were no significant interactions between comparison sequence and any other factor, suggesting that comparison sequence did not interfere with the effect of moon position, background brightness, or drawing.

\section{EXPERIMENT 3}

Experiment 3 sought to explore the role of the size of the standard moon and the type of distance cue (i.e., linear perspective and interposition) in the illusory distance component of the moon illusion in drawings.

\section{Method}

\section{Participants}

Forty-eight participants - 36 females and 12 males, ages 17 to 32 years $(M=19.58, S D=3.09)$-participated in Experiment 3. All participants had normal or corrected-to-normal vision and were naive to the purpose of the experiment. Each participant was randomly assigned to one of three groups corresponding to a particular standard moon size: small, medium, or large; each group was composed of 16 participants.

\section{Materials}

The experimental drawings used in Experiment 3 were consistent with those used in Experiment 2; however, the horizon line was omitted as a cue to distance, and all drawings possessed a black background. The combination of moon position and context resulted in eight different experimental drawings. The size of the moon in each drawing was varied, resulting in three standard moon size conditions: (1) small, in which the size of the standard moon was $58.56 \mathrm{~mm}$ in diameter (subtending a visual angle of $2.1^{\circ}$ ); (2) medium, in which the size of the standard moon was $61 \mathrm{~mm}$ in diameter (subtending a visual angle of $2.18^{\circ}$ ); and (3) large, in which the size of the standard moon was $63.44 \mathrm{~mm}$ in diameter (subtending a visual angle of $2 \cdot 27^{\circ}$ ). A separate set of experimental drawings was created for each standard moon size. All drawings were computer generated and displayed using an LCD projector.

\section{Procedure}

The method of paired comparisons, in which each stimulus image is used as the standard image, was employed in Experiment 3. Each of the eight experimental drawings was paired with each of the other experimental drawings, resulting in 28 pairs. Each of the 28 pairs was presented to each participant 10 times, for a total of $280 \mathrm{com}-$ parisons. In order to limit position and carryover effects, half of the 28 original pairs were randomly selected, and the drawings were in-
}

terchanged, so that the two images were presented on opposite sides. All pairs were randomized by E-Prime. In each trial, the participants were asked to indicate which stimulus moon (the one in the drawing displayed on the left or the one in the drawing displayed on the right) they perceived to be more distant. They indicated their selection by pressing the "Z" key on a keyboard for the left-hand moon and the " $\mathrm{M}$ " key for the right-hand moon.

\section{Results}

The proportion of times that each participant judged the moon in the drawing on the left to be farther away, as a function of image pair, was calculated and served as the dependent variable. Each proportion was then converted to a $z$ score as a means to standardize these values (see Torgerson, 1963). The $z$ scores were interpreted at face value; each proportion above .50 corresponded to a positive $z$ score, and each proportion below .50 corresponded to a negative $z$ score. The higher the $z$ score, the farther away the moon was judged. Mean $z$ scores for all conditions are presented in Figure 7.

The analysis revealed that participants judged the elevated moon to be farther away than the horizon moon overall in all drawing conditions for the large and small moon-size groups (Figure 7). This was consistent in the medium moon size group except for the control drawing. The moon was also perceived to be farther away overall in the linear perspective alone and in the linear-perspectiveand-interposition-together conditions than in the interposition alone conditions. Overall, as the number of depth cues increased, the apparent distance of the moon increased.

\section{Discussion}

The control images used in Experiment 3 consisted of the moon either in its horizon or in an elevated position, embedded in a black background. Results indicated that trends were similar between the control figures and the other experimental drawings in the small and large standard moonsize groups; participants judged the elevated moon to be farther away than the horizon moon. However, the control condition for the medium size group did not follow the typical trend; participants judged the elevated moon to be closer than the horizon moon. Since this was the only condition in which results were counterintuitive, it was thought to be an anomaly. Regardless, this finding highlights the importance of the use of proper control stimuli.

The moon was judged farther away overall in the linear perspective alone and in the linear-perspective-andinterposition-together conditions than in the interpositionalone condition. Linear perspective is a strong cue to depth in that it creates a vanishing point that typically falls on the horizon line in an image (Weiten, 2007). By contrast, interposition may not suggest a horizon division, since it can occur above and below the horizon line. As such, it may be a more accurate cue from which to form relative distance judgments of the objects in an image (i.e., objects in front are closer than objects behind or that are partially occluded). With the addition of multiple distance cues, such as in the linear perspective and interposition condition, the visual system is provided with much more information about distance than it would have if any one distance cue 


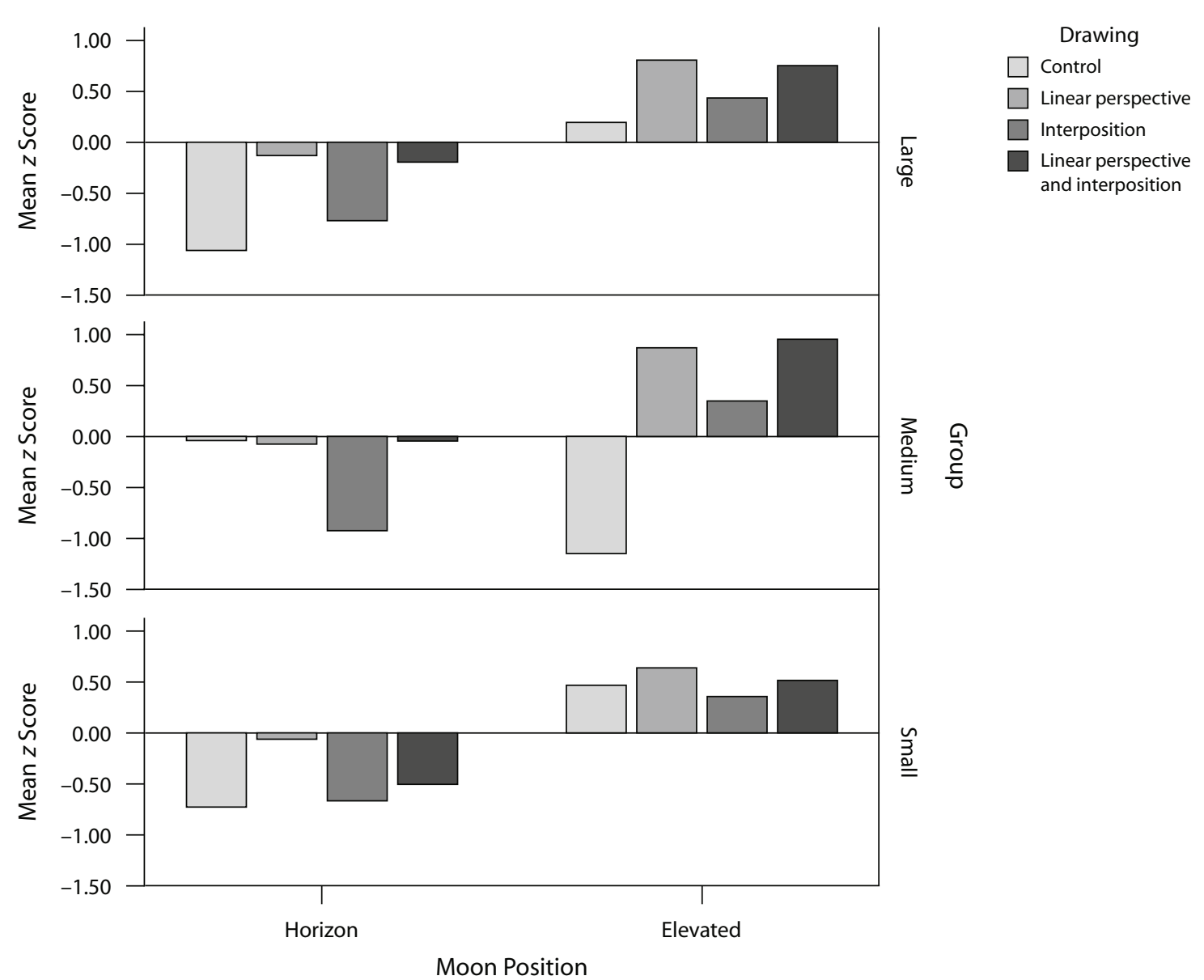

Figure 7. Mean $z$ scores broken down by drawing condition and group. Participants judged the elevated moon to be farther away than the horizon moon overall, in all conditions, except for the elevated control drawing for the medium group.

was presented alone; the addition of subsequent distance cues should create a greater impression of depth.

The moon illusion is often cited as an example of the size-distance paradox (Gruber, 1954); in addition to perceiving the horizon moon to be larger than the elevated moon, observers should also perceive the horizon moon to be farther away than the elevated moon. Rock (1975) distinguished between perceived or apparent distance and registered distance-registered distance being formed from information extracted from the environment by the visual system. This information may be gathered outside of conscious awareness and may not necessarily influence our judgments of distance. As a result of the two-dimensional nature of drawings, several cues to distance (e.g., accommodation, convergence) are eliminated from perception. A true judgment of distance, or registered distance, must be based on pictorial distance cues and the brain's interpretation of these distance cues, as opposed to other cognitive influences (e.g., the familiar size of objects). However, although the literature suggests a relationship between apparent size and apparent distance, it is not always clear as to which way the relationship is directed. Furthermore, others have also suggested that apparent size is assessed prior to apparent distance. The initial estimate of size may be based on biases or expectations and may disregard distance cues that contradict that bias (Kaufman et al., 2007). Kaufman et al. suggested the need for additional research using criterion-free judgments of apparent size.

\section{GENERAL DISCUSSION}

The present study included a series of three experiments that sought to explore the magnitude of the moon illusion in computer-generated drawings. Experiment 1 examined the number and salience of depth cues, as well as background brightness on the illusion. Experiment 2 examined the role of the horizon line, linear perspective, interposition, and background brightness, and Experiment 3 explored the role of linear perspective, interposition, and the size of the standard moon in the illusory distance component of the moon illusion.

Experiments 1 and 2 revealed that participants judged the horizon moon to be larger than the elevated moon and that the addition of subsequent distance cues had a greater effect on the apparent size of the horizon moon than on that of the elevated moon. Experiment 2 failed to support the role of the horizon line in affecting the size of the illusion. By contrast, linear perspective and interposition 
were found to be significant contributors to the magnitude of the illusion in drawings.

The results of the present experiments are consistent with the existing literature that has examined the relationship between apparent size and apparent distance as suggested by Emmert's law (Corum, 1976; Hershenson, 1989). In addition, these findings are consistent with the adjacency principle that suggests that the distance cues in the drawing will have a greater effect on the apparent size of the horizon moon than that of the elevated moon, due to their proximity to the horizon moon (Gogel, 1973).

The present experiments included control images to establish baseline measures of apparent size. The difference between participant size judgments obtained from the control stimuli and size judgments obtained from the experimental drawings indicated the relative effect of the distance cues on the apparent size of the moon in the two moon positions. For example, the results of Experiment 1 indicated a significant difference between the control image and all other experimental drawings. In Experiment 2, no significant difference was found between the control stimulus and the horizon-line-only stimulus.

Experiments 1 and 2 also revealed that participants judged the experimental moon to be larger overall in the black background conditions than in the white and gray background conditions. This finding emphasizes the importance of ecological validity when using drawings as a means to explore the factors contributing to this illusion. This finding is consistent with past research that has suggested a relationship between apparent brightness and apparent size (e.g., Holway \& Boring, 1940; Robinson, 1954), and this result highlights the importance of exploring the moderated relationship between apparent brightness, apparent size, and apparent distance; apparent brightness and apparent distance may be influencing participants' perceptions of the size of the moon.

Experiment 3 provided additional support for the illusory distance component of the moon illusion, as well as the use of drawings as an appropriate method to explore this component of the moon illusion. Participants judged the elevated moon to be farther away than the horizon moon in all conditions except for one. Furthermore, consistent with past literature (e.g., Coren \& Aks, 1990), the number of depth cues also played a role in participants' judgments of distance. The moon was judged to be farther away overall in the linear perspective and in the linear-perspective-and-interposition conditions than in the interposition-alone conditions.

Overall, the results of the present study support the use of drawings as a viable means to investigate the moon illusion. The present study contributes to the existing literature that has examined the moon illusion in images, provides additional support for the role of depth cues as a primary contributor to the illusion, and has provided novel findings on the role of background brightness and the horizon line in the magnitude of this illusion.

A logical next step would be to present observers with two moons of different sizes and to ask them to indicate which moon appears closer. If large moons appear closer than small moons, then this would be consistent with the size-distance paradox. If observers do not judge larger moons to be closer, then alternative explanations for this illusion may be considered (e.g., familiar size; Epstein, 1965). A follow-up experiment may determine whether participants consistently judge the elevated moon to be farther away, regardless of its size in comparison with the horizon moon.

\section{AUTHOR NOTE}

Address correspondence to S. A. H. Jones, Psychology Department, University of New Brunswick, Saint John, P.O. Box 5050, Saint John, NB, Canada E2L 4L5 (e-mail: sahj0812@yorku.ca).

$$
\begin{array}{r}
\text { Note-Accepted by the previous editorial team, } \\
\text { when Thomas H. Carr was Editor. }
\end{array}
$$

\section{REFERENCES}

BorIng, E. G. (1943). The moon illusion. American Journal of Physics, 11, 55-60

Coren, S., \& AKs, D. J. (1990). Moon illusion in pictures: A multimechanism approach. Journal of Experimental Psychology: Human Perception \& Performance, 16, 365-380.

Corum, M. C. (1976). On the moon illusion. Santa Monica, CA: Rand.

ENNs, J. T. (2004). The thinking eye, the seeing brain: Explorations in visual cognition. New York: Norton.

Epstein, W. (1965). Nonrelational judgments of size and distance. American Journal of Psychology, 78, 120-123.

Gogel, W. C. (1973). The organization of perceived space: II. Consequences of perceptual interactions. Psychologische Forschung, 36, 223-247.

GoldSTEIN, E. B. (2007). Sensation and perception: Instructor's edition (7th ed.). Belmont, CA: Thomson Wadsworth

Gruber, H. E. (1954). The relation of perceived size to perceived distance. American Journal of Psychology, 67, 411-426.

Helson, H. (1964). Adaptation-level theory: An experimental and systematic approach to behavior. New York: Harper \& Row.

Hershenson, M. (ED.) (1989). The moon illusion. Hillsdale, NJ: Erlbaum.

Holway, A. H., \& Boring, E. G. (1940). The dependence of apparent visual size upon illumination. American Journal of Psychology, 53, 587-589

Kaufman, L., \& Rock, I. (1962). The moon illusion. Scientific American, 207, 120-132.

Kaufman, L., Vassiliades, V., Noble, R., Alexander, R., KaufMAN, J., \& EDLUND, S. (2007). Perceptual distance and the moon illusion. Spatial Vision, 20, 155-175.

KING, W. L., \& GRUber, H. E. (1962). Moon illusion and Emmert's Law. Science, 135, 1125-1126.

Mandes, E. (1985). Interposition and size constancy: A developmental study. Perceptual \& Motor Skills, 61, 196-198.

Over, R. (1962). Brightness judgments and stimulus size and distance. British Journal of Psychology, 53, 431-438.

Pressey, A. W. (1967). A theory of the Mueller-Lyer illusion. Perceptual \& Motor Skills, 25, 569-572.

REDDING, G. M. (2002). A test of size-scaling and relative-size hypotheses for the moon illusion. Perception \& Psychophysics, 64, 1281-1289.

Robinson, E. J. (1954). The influence of photometric brightness on judgments of size. American Journal of Psychology, 67, 464-474.

Rock, I. (1975). An introduction to perception. New York: Macmillan.

Rock, I., \& KaUfMAN, L. (1962). The moon illusion, II: The moon's apparent size is a function of the presence or absence of terrain. Science, 136, 1023-1031.

Torgerson, W. S. (1963). The law of comparative judgment. In W. S. Torgerson (Ed.), Theory and methods of scaling (pp. 159-204). New York: Wiley.

Watson, W. A. (1957). Contrast, assimilation, and the effect of central tendency. American Journal of Psychology, 70, 560-568.

Weiten, W. (2007). Psychology: Themes and variations (7th ed.). Belmont, CA: Thomson Wadsworth.

(Manuscript received May 9, 2007; revision accepted for publication July 15, 2008.) 